\title{
Gain Antenna Measurement using Single Cut Near Field Measurements
}

\author{
M. Sierra-Castañer,
}

\author{
F. Saccardi, L.J. Foged
}

\begin{abstract}
Some antennas require rapid validation at a reduced measurement distance while maintaining sufficient accuracy in the determination of pertinent antenna parameters such as gain. In particular, for cellular base station antennas in production phase the measurement time can be a limitation. In these cases, a rapid check of the radiation performance in the two main planes is sufficient. Other examples are phase arrays with high degree of steering that would require considerable measurement time for characterizing all steering positions.
\end{abstract}

This paper presents a near-field antenna test procedure providing single or double main plane patterns including the gain. The procedure is applicable to antennas, with separable excitation in the two main planes. The test set-up is based on an azimuth positioner and near to far-field transformation based on expansion in cylindrical modes. The paper shows results for gain measurements. Near to far-field transformation is performed using the cylindrical modes expansion assuming a zero-height cylinder. This allows the use of a FFT in the calculation of the far field pattern including probe correction. In the case of gain, the near to far-field transformation factor is calculated for bore sight direction, taking advantage of the separable excitation properties of the antenna. This factor is used in the gain calculation by comparison technique.

\section{INTRODUCTION}

In the literature, different methods for reducing antenna measurement time have been explored. One of these techniques is single cut near field to far field transformation. For single cut, near to far-field transformation, one of the first methods is the discrete beam sampling method (DBSM), and it is explained in [1-3]. That algorithm is based on the Fourier series decomposition of the $1 / \mathrm{R}^{2}$ term of the field propagation in spherical coordinates, and it is valid for Fresnel Zone Measurements, where the $1 / \mathrm{R}^{3}$ term can be neglected. Technical University of Madrid (UPM) implemented this method for the measurements of the ASAR panels of the ENVISAT satellite [4] in the two main planes of the forward hemisphere $\left(-90^{\circ}<\theta<90^{\circ}\right)$. Later, it was adapted for the measurement of the two main planes and gain of BTS antennas in the whole angular range by processing the measurements in two halves and then combining the results [5]. One of the limitations of this algorithm is the absence of probe correction.

F. Las Heras proposed in [6] another algorithm based on a least-squares algorithm to include probe correction for the same application. Also, in [7], F. Las Heras solved the problem of the calculation of the main plane far-field radiation with a method based on the reconstruction of equivalent magnetic currents (EMCs) using decoupled integral equations and onedimensional source components.

The authors presented the algorithm used in this paper for the calculation of the radiation pattern in [8]. This algorithm is based in the cylindrical mode decomposition of the measurements on a ring, considered as a cylinder of zero height, and the fundamentals are explained in the next section.

R. Cornelius et al. presented in [9] the comparison of results using three different algorithms: this last algorithm, the one based on the Fourier series decomposition and one implemented by R. Cornelius based on the spherical wave expansion of the measurement in one cut. That paper analysed the advantages and drawbacks of each of the three methods, concluding that the method based on cylindrical mode expansion was more appropriate since it could work directly with the full ring and included probe correction. That paper only presented the antenna radiation patterns, without gain determination. This paper shows the method for the antenna gain calculation for one or two cuts and shows measured results for some specific antennas.

The paper is structured in this way: section 2 presents the algorithms for single or double cuts gain determination using single cut near to far field transformation. Section 3 presents the validation of the method through results of the measurement of different antennas (horns, base station arrays, reflectors) to analyze the limitations of the algorithm. Section 4 includes the conclusions and the possible implementation in a production test system.

\section{Algorith FOR ANTENNA GAIN CALCUlation}

This section explains briefly the fundamentals of the single cut to $\mathrm{NF} / \mathrm{FF}$ transformation and gain calculation algorithm. The algorithm is based on the cylindrical NF/FF transformation [10], but applied to a zero height cylinder. In that sense, any of the rings of the sphere can be considered as a zero height cylinder. Since only one spatial dimension is considered, the complexity of the algorithm is reduced. However, probe correction works in the same way of the classical cylindrical $\mathrm{NF} / \mathrm{FF}$ transformation. The work presented in [8] explained the fundamentals of this single cut NF/FF transformation. The presented results were satisfactory for the measurement of the main planes of antennas with separable excitation. 
Figure 1 shows, as an example, the measurement of the FF for one single cut of an X-band slotted array antenna having a diameter of approximately $31 \mathrm{~cm}$. The measurement of only the E-plane of the antenna was performed at $9.3 \mathrm{GHz}$ at and at measurement distance of 2 meters. The FF distance for this antenna is approximately 6 meters at $9.3 \mathrm{GHz}$. In red, it is shown the results using this method, in blue using conventional spherical NF/FF transformation technique [11-12]. As it can be observed, the results agree pretty well for both the co-polar and cross-polar radiation pattern.
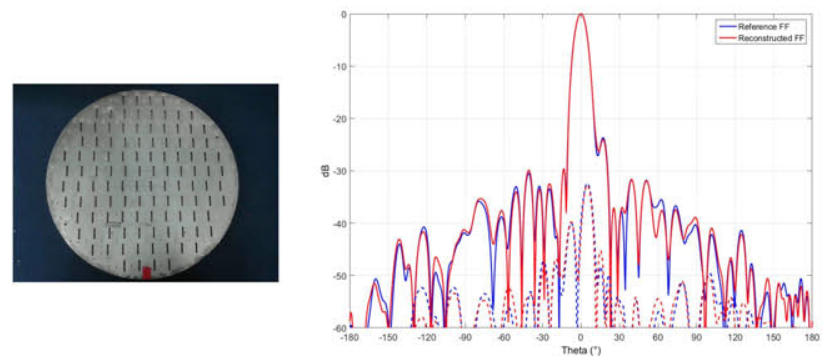

Figure 1. Application of single cut NF/FF transformation to the E-plane of a slotted array sntenna.

The gain can be calculated through the NF substitution technique using a Standard Gain Horn (SGH) which gain has been already calibrated (1)

$$
\mathrm{G}_{\mathrm{AUT}}(\mathrm{dBi})=\mathrm{G}_{\mathrm{SGH}}(\mathrm{dBi})+\mathrm{P}_{\mathrm{AUT}, F F, \varphi=\varphi \mathrm{o}}(\mathrm{dB})-\mathrm{P}_{\mathrm{SGH}, F F, \varphi=\varphi \mathrm{o}}
$$

where $G_{\text {AUT }}$ is the gain of the Antenna Under Test (AUT), $G_{S G H}$ is the gain of the $\mathrm{SGH}$, and $\mathrm{P}_{\mathrm{AUT}, \mathrm{FF} / \mathrm{SGH}, \mathrm{FF}}$ are the received power at a specific angular direction using either AUT either $\mathrm{SGH}$. Since, the measurement is carried out in $\mathrm{NF}, \mathrm{P}_{\mathrm{AUT}, \mathrm{FF}, \varphi=\varphi \mathrm{O}}$

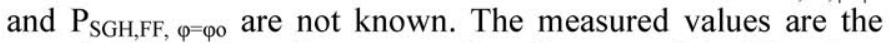
ones in NF and can be expressed as the sum of the FF values and a NF/FF transformation factor as reported in (2)

$$
\mathrm{P}_{\mathrm{AUT} / \mathrm{SGH}, \mathrm{NF}, \varphi=\varphi \mathrm{O}}(\mathrm{dB})=\mathrm{P}_{\mathrm{AUT} / \mathrm{SGH}, \mathrm{FF}, \varphi=\varphi \mathrm{O}}(\mathrm{dB})+\mathrm{Ww}_{\mathrm{AUT} / \mathrm{SGH}}
$$

The NF/FF factor accounts for the modification of the AUT pattern when transforming from NF to FF and it can be computed as the difference between the FF and NF directivity in a specified direction (e.g. theta $=0$ ).

If the FF pattern is normalized maintaining constant the power radiated through the ring in both NF and FF, the NF/FF transformation factor becomes close to zero when the measurement is performed in FF. Therefore, in the case of the $\mathrm{SGH}$, and for most of the cases, this factor can be neglected, making the measurement process faster.

In the case of the AUT, it is assumed that the excitation in the antenna aperture is separable. Therefore, the field can be expressed as a multiplication of two factors, each one depending on the aperture excitation on each main plane. In the same way, the NF/FF factor can therefore be expressed as the product of the NF/FF factor on each main plane. Also in the case of linear arrays, or thin apertures, it can be considered that the NF/FF factor in the thin side is also close to zero. In this case, only one cut would be necessary to characterize the gain of the AUT. The key point of this procedure is thus the calculation of this NF/FF factor.

Figure 2 shows the calculation of the NF/FF factor at difference measurement distances for the slotted array shown in Figure 1. Red trace shows the NF/FF factor calculated from full sphere; blue trace shows the one computed as the product of the NF/FF factor for the two main cuts. The green curve shows instead the difference between the other two traces. Good agreement is obtained for this antenna if the measurement is not carried out at an extreme NF distance.

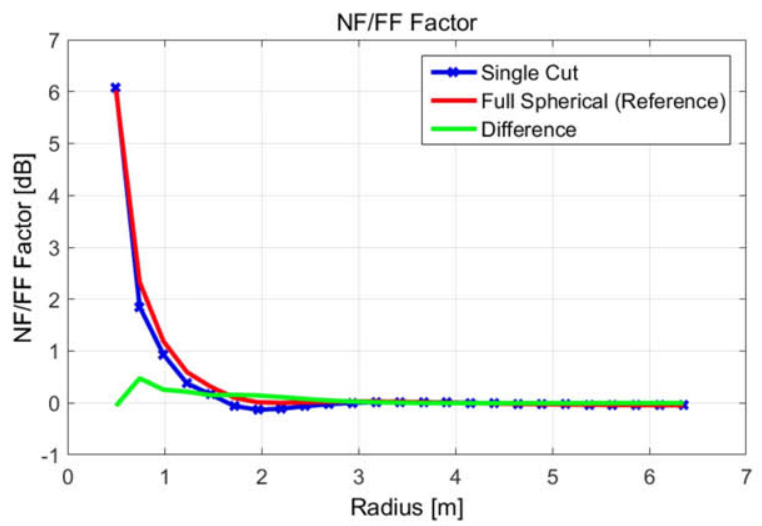

Figure 2. Comparison of the NF/FF factor for a planar slotted array calculated through the information of the full sphere or the product of the two main planes.

\section{APPLICATION TO DIFFERENT ANTENNAS}

This section shows the validation of the gain calculation algorithm for different cases. The antennas have been measured in the facilities of LEHA-UPM [13]. The results are compared with the gain calculated using Spherical NF/FF transformation software [12] and full spherical acquisition and also with measurements performed in other ranges (where possible). Three different antennas have been tested; some of them have been measured in NF, others in Fresnel zone and some of them close to far field distance.

\section{A. Gain measurement results for BTS1940 antenna.}

The first antenna is the MVI BTS1940 antenna [14] which dimensions are $1400 \times 200 \mathrm{~mm}$. This antenna is a linear array used as reference antenna to evaluate the quality of base station antenna measurement systems. Figure 3 shows the antenna itself and the comparison of the results of the gain when the antenna has been measured in Fresnel Zone (5.35 meters), while the FF distance is between 21 and $31 \mathrm{~m}$, depending on the frequency. Single or dual cut slightly overestimate the gain, but the difference is bounded in $0.5 \mathrm{~dB}$ using one cut (violet trace) and $0.4 \mathrm{~dB}$ using two cuts (green trace) in the worst case. 

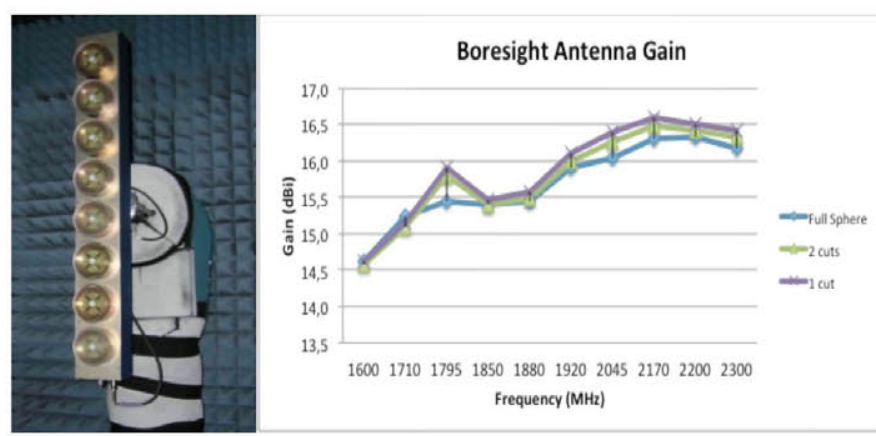

Figure 3. Comparison of gain measurements for BTS1940 antenna.

\section{B. Gain measurement results for SH2000 antenna.}

The second antenna is the MVI dual ridge horn antenna SH2000 [14] working in the frequency band from 2 to $32 \mathrm{GHz}$. The overall dimensions are $62.4 \times 108 \times 103.7 \mathrm{~mm}$. This antenna is a standard gain horn based on a ridged design combining stable gain performance and low VSWR with ultra-wide band frequency operation. The horn is single linear polarized with excellent cross-polar discrimination, ideal for gain calibration of antenna measurement systems or as wideband probes in classical FF test ranges. The antenna has been measured at the LEHA-UPM spherical near field system, at a measurement distance of 3.86 meters, in the frequency band from 18.2 to 31 $\mathrm{GHz}$ for this exercise. For these frequencies, the FF goes from 1.9 to $3.2 \mathrm{~m}$, therefore the antenna is in FF. In any case, it is observed in Figure 4 that the double cut NF/FF transformation (green trace) corrects the small error occurred when finite FF distance (violet trace) is used (between 0.1 and $0.2 \mathrm{~dB}$ in this case depending on the frequency).
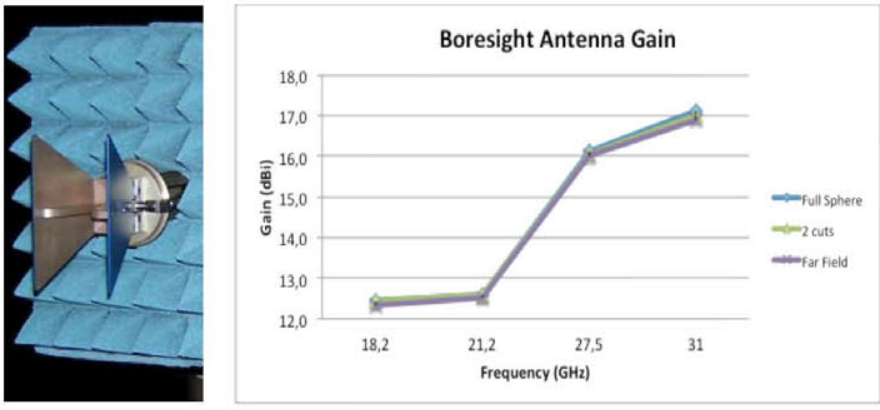

Figure 4. Comparison of gain measurements for SH2000 antenna.

\section{Gain measurement results for SR40 antenna.}

The third antenna is the MVI SR40 offset reflector antenna [14]. The dimensions of the antenna are $561 \times 400 \times 315 \mathrm{~mm}$. It is fabricated with Aluminium, and excited with the SH2000 feeder $(2-32 \mathrm{GHz})$. The antenna has been measured in the frequency band from 10.7 to $38 \mathrm{GHz}$, at a distance of $5.45 \mathrm{~m}$. The far field distance is from 33 to $120 \mathrm{~m}$ at those frequencies, therefore, the measurement is clearly performed at a NF distance.
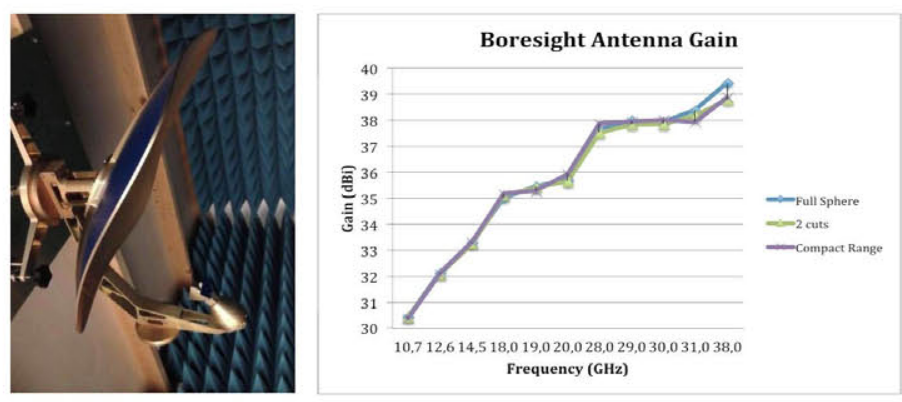

Figure 5. Comparison of gain measurements for SR40 antenna.

Figure. 5 shows the gain measurements using full sphere NF method (blue trace), the two cuts method (green trace) and the measurement in a compact range (violet trace). In the worst case, the difference between full sphere and 2 cuts is $0.6 \mathrm{~dB}$ (at $38 \mathrm{GHz}$ ). However, even in this case, the 2 cuts agrees pretty well with the measurement in compact range. For the other cases, the difference between full sphere and 2 cuts in less than $0.2 \mathrm{~dB}$

\section{CONCLUSIONS}

Single cut NF/FF transformation has been applied in the gain measurement of different separable excitation antennas using substitution technique. In the case of a BTS antenna (linear array), the method can be applied with only information of the main cut (vertical pattern). In the case of the other two antennas (the horn and offset reflector) the NF/FF transformation factor, and therefore, the gain, can be calculated through the measurement and individual processing of the $\mathrm{NF} / \mathrm{FF}$ transformation factor in the two main cuts.

The measurements of these three antennas, performed in different measurement scenarios (Fresnel Zone, $\mathrm{FF}$ at a finite distance and NF) conclude that this algorithm improves the results with respect FF measurement and gets enough accurate results (comparable to other measurement systems as compact range) and the uncertainty is bounded into a reasonable value. Future works will emphasize in the limitations of this algorithm and the performance depending on the measurement distance.

The main application for this work is the possibility of making very fast measurement in the production phase of commodity antennas. Antenna gain and pattern in the main pattern cuts can be characterized (almost) in real time using this method in combination with a multiprobe system.

\section{ACKNOWLEDGEMENTS}

This work has been done thanks to the collaboration between MVI and UPM, the Spanish project ENABLING5G "Enabling Innovative Radio Technologies for 5G networks" (TEC2014-55735-C3-1-R), from the Research Plan of the Spanish Government, and the project from Madrid Region Government S2013/ICE-3000 (SPADERADAR-CM). 


\section{REFERENCES}

[1] K. Wu and S. Parekh. "Methods of transforming antenna Fresnel region fields to far region fields". Proc. AMTA Monterrey, CA. October 1989. Pp. 11.9 to 11.14

[2] Marlborg, L. Hansson. "Quasi far-field to far field transformation". Proc. 11th ESTEC Workshop on Antenna Measurements, June 1988.

[3] Evans, Gary E. "Antenna Measurement Techniques". Artech House, 1990.

[4] F. Las Heras, B. Galocha, J.L. Besada. "ERS-B Measurement Using a Spherical Near-Field and Fresnel Techniques at ETSIT of Madrid Facility". Madrid, Spain, Ref: UPM/ETSIT/DSSR/GR/06/95

[5] M. Sierra Castañer, S. Burgos, "Fresnel Zone to Far-field Algorithm for Rapid Array Antenna Measurements", Proceedings of the 5th European Conference on Antennas and Propagation (EUCAP), Rome 2011, pp. 3251-3255.

[6] F. Las-Heras, B. Galocha, J.L. Besada A Method to transform measured Fresnel patterns to far-field based on a least-squares algorithm with probe correction" AMTA, 17th Annual Meeting \& Symposium, 1995. Virginia, AMTA Proc. Pp. 352-357

[7] F. Las-Heras, B. Galocha, J.L. Besada. Far-Field Performance of Linear Antennas Determined from Near-Field Data. IEEE Trans. On Antennas and Propagation. Vol. 50, no.3, March 2002, pp.408-410.
[8] T. Salmerón-Ruiz, M. Sierra-Castañer, F. Saccardi, S. Burgos, F. J. Cano-Fácila, Lars J. Foged. "A Fast Single Cut Spherical Near-Field-toFar-Field Transformation Using Cylindrical Modes". Proceedings of the VIII European Conference on Antennas and Propagation, EuCAP2014, Den Haag, April 2014

[9] R. Cornelius, T. Salmerón-Ruiz, F. Saccardi, L. Foged, D. Heberling, and M. Sierra-Castañer. "A Comparison of Different Methods for Fast Single-Cut Near-to-Far-Field Transformation" IEEE Antennas and Propagation Magazine, vol. 56, no. 2, April 2014, pp. 252-261.

[10] W. M. Leach and D. T. Paris, "Probe compensated near-field measurements on a cylinder", IEEE Transactions on Antennas and Propagation, vol. 21, no. 4, pp. 435-445, Jul. 1973.

[11] J. E. Hansen, "Spherical Near-Field Antenna Measurements". London, UK: Peter Peregrinus LTd., 1988.

[12] SNIFTD. www.ticra.com

[13] LEHA-UPM. www.gr.ssr.upm.es/leha

[14] Microwave Vision Group. www.microwavevision.com 